\title{
Thiophene-Based Trimers for In Vivo Electronic Functionalization of Tissues
}

\author{
Daniele Mantione, ${ }^{*}, \|$ Emin Istif, $\|$ Gwennael Dufil, Lorenzo Vallan, Daniela Parker, Cyril Brochon, \\ Eric Cloutet, Georges Hadziioannou, Magnus Berggren, Eleni Stavrinidou,* and Eleni Pavlopoulou*
}

Cite This: ACS Appl. Electron. Mater. 2020, 2, 4065-4071

Read Online

ACCESS

Llll Metrics \& More

Article Recommendations

Supporting Information

ABSTRACT: Electronic materials that can self-organize in vivo and form functional components along the tissue of interest can result in a seamless integration of the bioelectronic interface. Previously, we presented in vivo polymerization of the conjugated oligomer ETE-S in plants, forming conductors along the plant structure. The EDOT-thiophene-EDOT trimer with a sulfonate side group polymerized due to the native enzymatic activity of the plant and integrated within the plant cell wall. Here, we present the synthesis of three different conjugated trimers based on thiophene and EDOT or purely EDOT trimers that are able to polymerize enzymatically in physiological $\mathrm{pH}$ in vitro as well as in vivo along the roots of living plants. We show that by modulating the

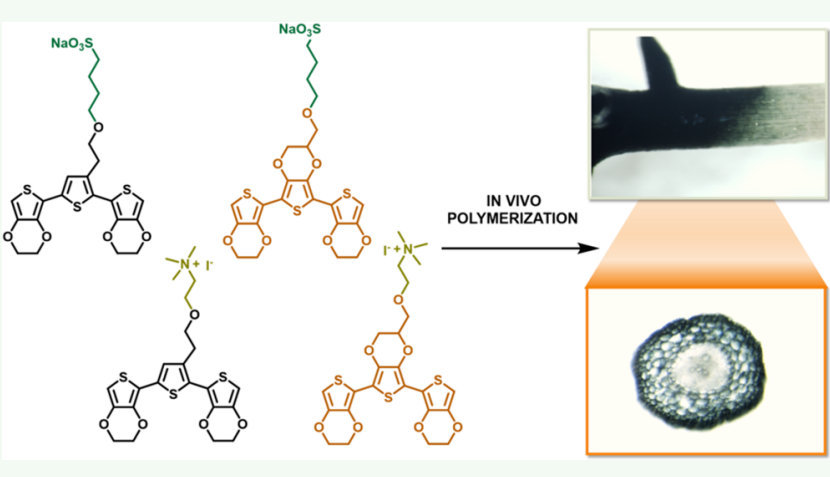
backbone and the side chain, we can tune the electronic properties of the resulting polymers as well as their localization and penetration within the root. Our work paves the way for the rational design of electronic materials that can self-organize in vivo for spatially controlled electronic functionalization of living tissue.

KEYWORDS: EDOT, conducting polymers, enzymatic polymerization, plant-mediated polymerization, bioelectronics, tissue engineering

\section{INTRODUCTION}

The interplay between electronic and ionic conduction in conjugated polymers makes them efficient transducers in bioelectronic devices where ionic signals are translated to electronic readout and vice versa. ${ }^{1-3}$ In addition, the soft nature of conjugated polymers renders them compatible with the biological milieu and stable within it. ${ }^{4}$ Thiophene and its derivatives, especially 3,4-ethylenedioxythiophene (EDOT) have been widely explored in organic bioelectronics, while the commercially available poly(3,4-ethylenedioxythiophene):polystyrene sulfonate (PEDOT:PSS) has been the benchmark material in many devices such as neural probes, ${ }^{5,6}$ biosensors, ${ }^{7,8}$ and electronic scaffolds for cell culture. ${ }^{9}$ While traditionally the bioelectronic interface is being built independently of the tissue, recent work highlights the possibility of developing the interface directly on the tissue with examples reported both in animals, for modulating neural activity, and in plants, for energy applications. Martin and coworkers were the first to demonstrate this concept by electropolymerization of EDOT directly in the brain of a mouse. $^{10,11}$ Recently, Bao and co-workers took another approach where they genetically modified neuronal cells to express enzymes that then polymerized aniline dimers directly onto the cell surface. ${ }^{12}$ Whereas in animals, so far, an either physical or chemical trigger is required for the polymerization of the conductor, we have shown that in plants the native physicochemical environment is sufficient to self-organize poly(4-(2,3-dihydrothieno[3,4-b]-[1,4]dioxin-2-yl-methoxy)1 -butanesulfonic acid, sodium salt) (PEDOT-S) $)^{13}$ and polymerize bis[3,4-ethylenedioxythiophene]-3-thiophene butyric acid, sodium salt, trimer (ETE-S), ${ }^{14}$ forming conductors integrated into the plant structure. By investigating the mechanism, we revealed that the presence of the peroxidase enzymes in the plant and the native concentration of hydrogen peroxide were sufficient for the enzymatic polymerization of the ETE-S trimer and for the integration of the polymer into the plant cell wall. ${ }^{15}$ Enzymatic polymerization of conjugated polymers has been demonstrated in in vitro conditions primarily at low $\mathrm{pH}$ for aniline, ${ }^{16,17}$ as well as for EDOT. ${ }^{18,19}$ So far, the ETE-S trimer is the only example of an enzymatically polymerized conjugated molecule in physiological pH. Importantly, ETE-S, being a water-soluble thiophene-based trimer, allows the preparation of stable and ready-to-use solutions and suites better biological applications than aniline and its derivatives. Moreover, the trimeric

Received: September 30, 2020

Accepted: November 16, 2020

Published: December 1, 2020 

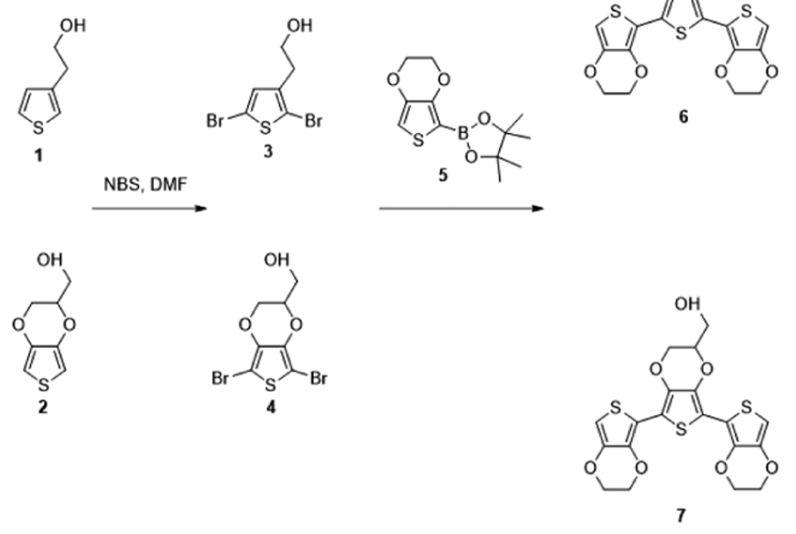

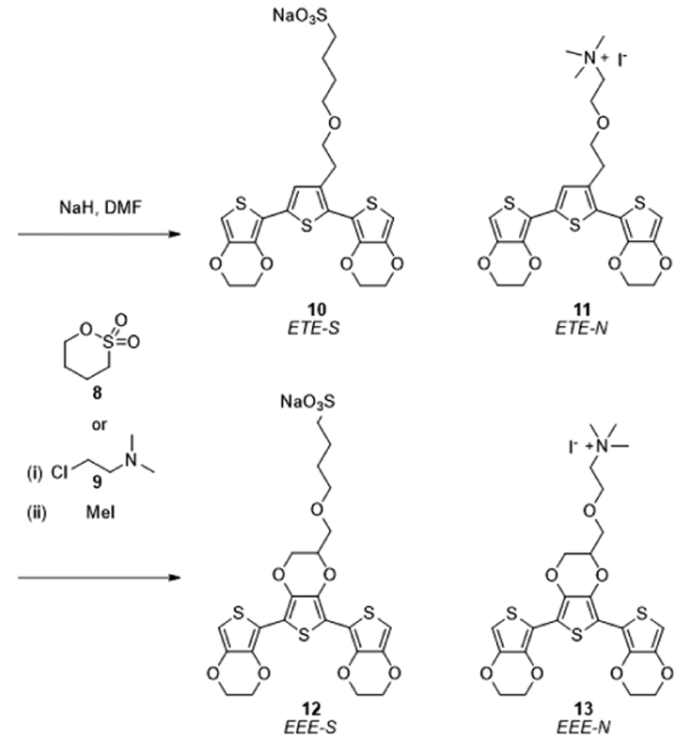

\section{RESULT AND DISCUSSION}

The synthesis of trimers 10-13 (Scheme 1) was achieved following a customizable route that involves few synthetic steps and results in improved yields with respect to synthetic routes previously reported for EDOT-based molecules. ${ }^{14}$ As far as ETE-S (10, Scheme 1) is concerned, it has been previously synthesized applying four synthetic steps: di-bromuration of the thiophene scaffold, propane sultone addition, and Suzuki reaction with a monoboronic acid EDOT derivative (Supporting Information, Scheme S7). ${ }^{14}$

This strategy is specific to ETE-S, and the as-prepared trimer is not suitable for further modifications, since the linkable hydroxy group has been converted into the sulfonate derivative during the first step. However, if the hydroxyl function of the thiophene ring is kept active, and a trimer-OH molecule is obtained, this trimer can be easily functionalized in a later step. $^{21}$ This strategy is extremely beneficial since further chemical modification can be performed directly on the trimer rather than on the initial monomer, thus reducing the synthetic steps needed for the generation of trimers with different functional groups. Starting from 3-(2-hydroxyethyl)thiophene (1), the hydroxyl-functionalized ETE trimer is obtained (6). Starting from hydroxymethyl EDOT (2) and its dibrominated derivative (4), we prepared the all-EDOT alter ego of ETE$\mathrm{OH}$, i.e., EEE-OH (7).

Next, two synthetic routes were pursued to introduce negative or positive charges on trimers 6 and 7 through the hydroxyl groups. By reaction with butane sultone, 6 and 7 were converted into their negatively charged sulfonate forms $\mathbf{1 0}$ (ETE-S) and 12 (EEE-S) in acceptable yields. To obtain their positively charged counterparts, 6 and 7 were mixed with 2chloro- $N, N$-dimethylethylamine for the introduction of a tertiary amine, as described in the literature for EDOT derivatives. $^{20}$ Next, an excess of iodomethane was used to convert the amine into its stable quaternized salt, resulting in the two cationic derivatives 11 (ETE-N) and 13 (EEE-N). The structures of trimers 10-13 were confirmed by proton and carbon-13 nuclear magnetic resonance $\left({ }^{1} \mathrm{H} /{ }^{13} \mathrm{C}\right.$ NMR) spectroscopies, heteronuclear single quantum correlation (HSQC) two-dimensional (2D) NMR spectroscopy, and attenuated total reflection (ATR) Fourier transform infrared 
Scheme 2. Scheme of Synthetic Pathways of the Polymerization of the Four Trimers, Resulting in Polymers 14-17
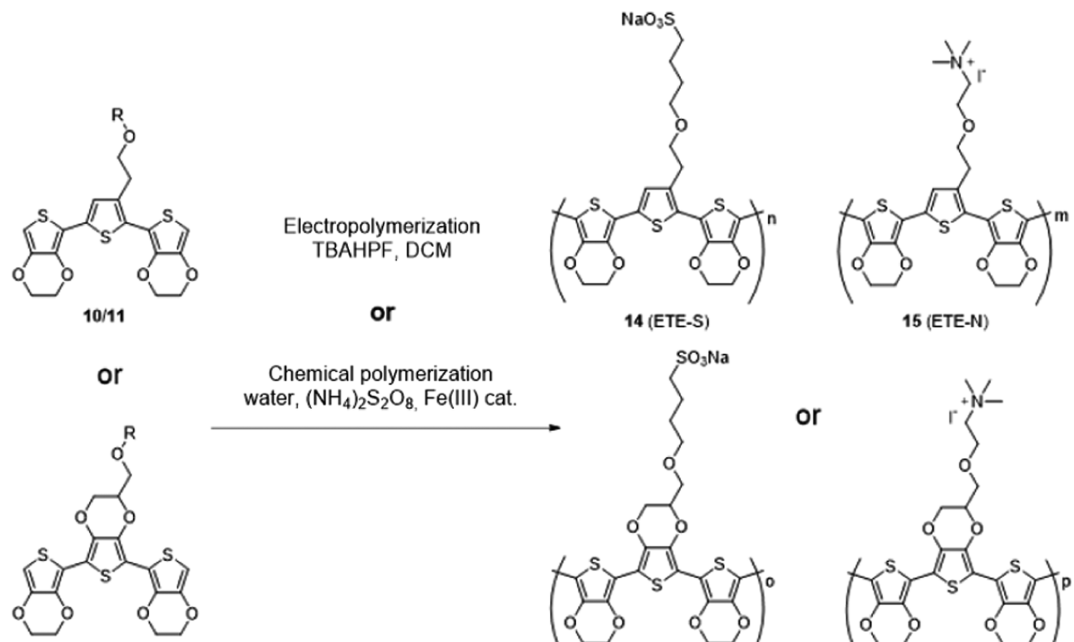

Chemical polymerization water, $\left(\mathrm{NH}_{4}\right)_{2} \mathrm{~S}_{2} \mathrm{O}_{8}$, $\mathrm{Fe}$ (III) cat.

$12 / 13$

$\mathrm{R}=\mathrm{nBu}-\mathrm{SO}_{3} \mathrm{Na} ; \mathrm{Et}_{-\mathrm{NMe}}{ }^{+} \mathrm{T}$

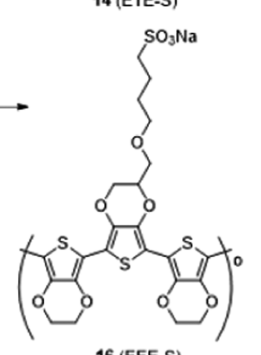

16 (EEE-S)

or

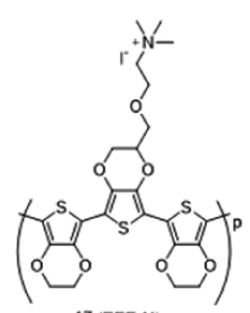

17 (EEE-N)

a.
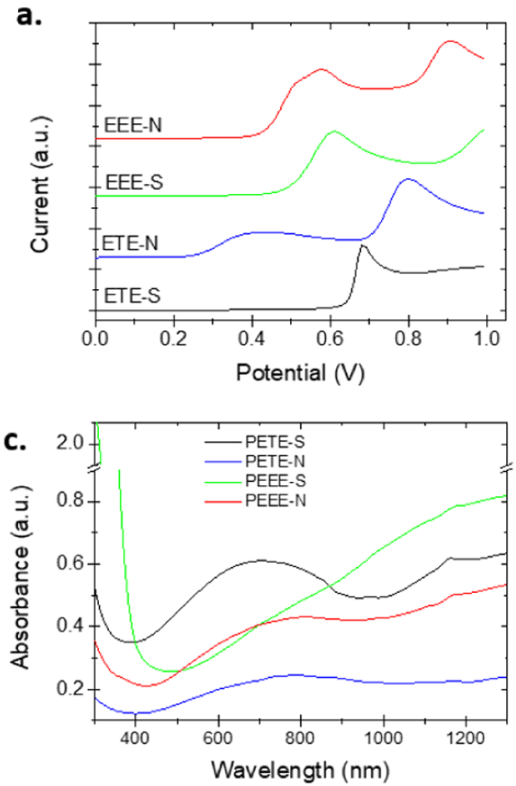

b.

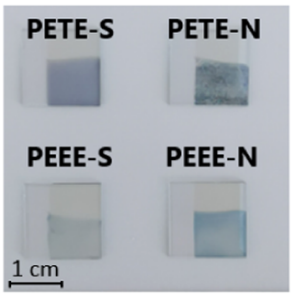

d.

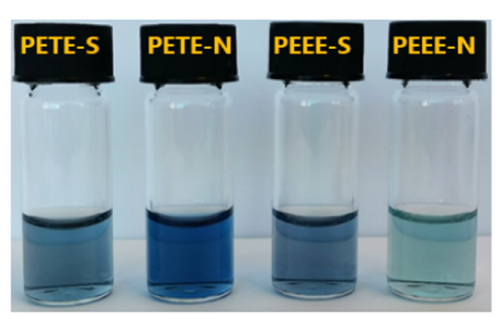

Figure 1. (a) Cyclic voltammetry curves recorded during the anodic scan of the first electropolymerization cycle of the four trimers. Curves are presented shifted along the current axis for clarity. Electropolymerizations were performed with $1 \mathrm{mM}$ solution in acetonitrile, using $0.1 \mathrm{M}$ of TBAHFP as the electrolyte. (b) Electropolymerized PETE-S, PETE-N, PEEE-S, and PEEE-N films, supported on ITO/glass. (c) Ultravioletvisible-NIR (UV-vis-NIR) absorption spectra of the chemically polymerized aqueous dispersions of PETE-S, PETE-N, PEEE-S, and PEEE-N. (d) The corresponding dispersions.

(FT-IR) spectroscopy. The data are presented in Supporting Information, Sections 2.4-2.7. Additionally, it is highlighted that all synthetic steps can be easily performed by nonsynthesis specialists, since the synthetic pathway mostly consists of operations of precipitation and filtration and only one column chromatography (after the Suzuki coupling reaction) is required.

The route described herein is highly reproducible, and it is believed to be a suitable and friendly tool for interdisciplinary researchers. Following synthesis, the four polymers were electrochemically, chemically, and enzymatically polymerized in vitro. Polymerization in living plant roots was induced via the native enzymes of the plants (Scheme 2). Electrochemical polymerizations to obtain polymers 14-17 (denoted hereafter as PETE-S, PETE-N, PEEE-S, and PEEE-N) were carried out using $0.1 \mathrm{M}$ of tetrabutylammonium hexafluorophosphate (TBAHFP) as an electrolyte in $1 \mathrm{mM}$ acetonitrile solution of the different trimers 10-13. To evaluate the potential electrodeposition of these molecules, cyclic voltammetry was performed on precoated indium tin oxide (ITO) glass substrates. During electropolymerization, an anodic potential in the range of $0-1 \mathrm{~V}$ was applied, and oxidation led to the growth of polymer films on ITO for all four trimers. The successful electrodepositions of the four monomers were confirmed by the constant increase in current between consecutive cycles (Figure S16), and they were completed after 10 cycles. Thin polymeric films were finally formed for all materials.

Figure 1a presents the anodic part of the first electropolymerization cycle of the four trimers. The two sulfonate- 
a.

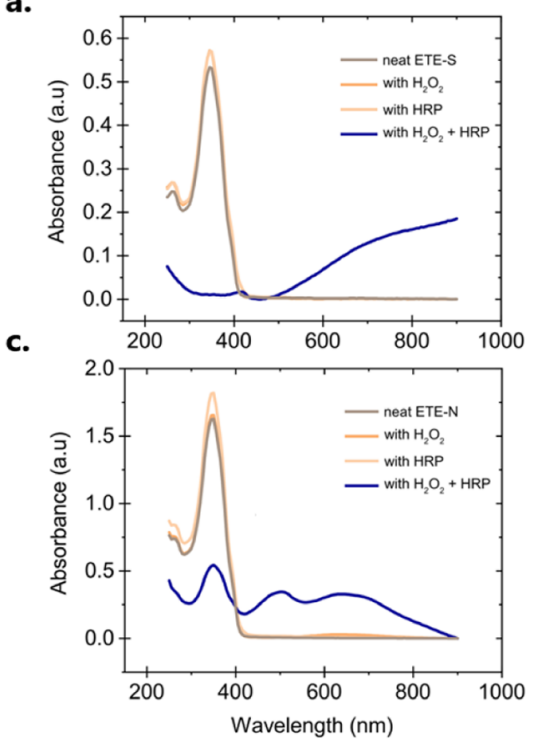

b.
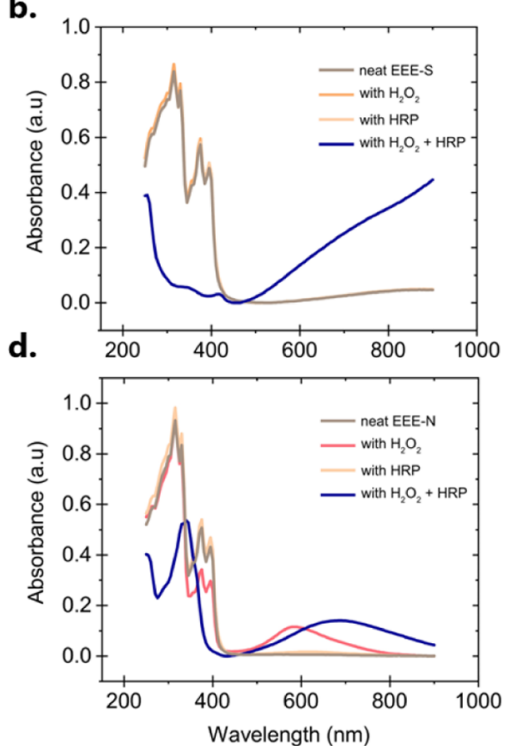

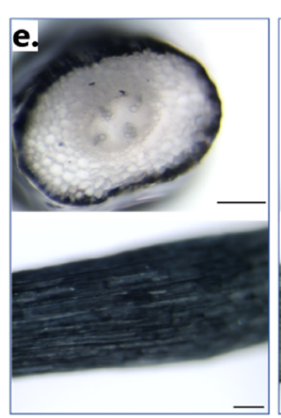

ETE-S

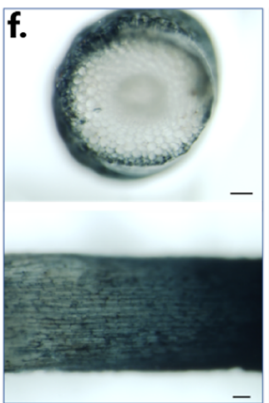

EEE-S

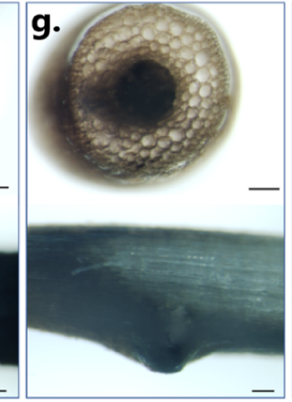

ETE-N

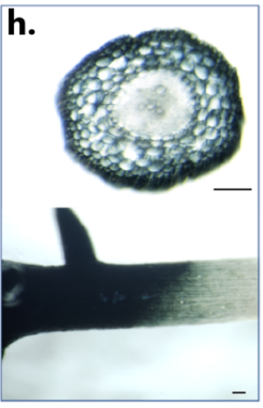

EEE-N

Figure 2. Enzymatic in vitro polymerization of (a) ETE-S, (b) EEE-S, (c) ETE-N, and (d) EEE-N. UV-vis spectra of neat trimer in DI water (360 $\mu \mathrm{M})$, trimer $(360 \mu \mathrm{M})$ in presence of hydrogen peroxide $(180 \mu \mathrm{M})$, trimer $(360 \mu \mathrm{M})$ in the presence of $\mathrm{HRP}\left(5 \mathrm{U} \mathrm{mL}^{-1}\right)$, and trimer $(360 \mu \mathrm{M})$ in the presence of both hydrogen peroxide $(180 \mu \mathrm{M})$ and $\operatorname{HRP}\left(5 \mathrm{U} \mathrm{mL}^{-1}\right)$ after $5 \mathrm{~min}$ of reaction. (e)-(h) Cross-sectional (top) and lateral views (bottom) of the bean roots in vivo functionalized for $24 \mathrm{~h}$ with $1 \mathrm{mM} \mathrm{mL}^{-1}$ of (e) ETE-S, (f) EEE-S, (g) ETE-N, and (h) EEE-N. The scale bar is $100 \mu \mathrm{m}$.

bearing trimers exhibit a single oxidation peak in the range of $0-1 \mathrm{~V}$, related to the oxidation of the external EDOT units of the trimers where chain elongation occurs. The oxidation peak recorded for EEE-S is located in a lower potential range with respect to ETE-S. The oxidation onset of EEE-S is at $0.47 \mathrm{~V}$ while that of ETE-S at $0.63 \mathrm{~V}$, indicating that EEE-S polymerizes more easily than ETE-S, as expected. ETE-N has two oxidation peaks, a very broad one located at low potentials (onset at $0.25 \mathrm{~V}$ ), which we attribute to the electrical compensation of the ammonium cations that energetically should arrive before the oxidation of the trimer's ends, and a sharper peak at $0.8 \mathrm{~V}$ (onset at $0.7 \mathrm{~V}$ ) that we attribute to the oxidation of ETE-N that drives its polymerization. Comparing the oxidation (polymerization) onsets of ETE-N and ETE-S, we conclude that the cationic moiety shifts the polymerization of ETE toward higher potentials. Concerning EEE-N, its voltammogram includes a double peak around $0.5 \mathrm{~V}$ and a single peak at $0.9 \mathrm{~V}$. Following the same reasoning as that for ETE-N, the oxidation of EEE-N to PEEE-N should be represented by the high potential peak. These multiple peaks are in agreement with the previously reported literature on polythiophene derivatives and indicate that the introduction of chemical functional groups can lead to additional oxidation reactions. ${ }^{22-24}$ Note that the oxidation potential of bare EDOT is $1.4 \mathrm{~V},{ }^{25}$ much higher than the potentials recorded for the four trimers, which is justified by the larger extension of conjugation in the case of trimers.

Figure $1 \mathrm{~b}$ presents the representative photos of the electrodeposited films, which appear blue on ITO. The surface morphology was imaged by means of atomic force microscopy (AFM) (Supporting Information, Figure S19). The surface of the PETE-S, PEEE-S, and PEEE-N films appears quite homogeneous and with low roughness, while PETE-N exhibits large protrusions.

The oxidative polymerization of the four trimers to derive polymers 14-17 was performed using ammonium persulfate as the oxidant and iron(III) as the catalyst. The polymerization was carried out for $18 \mathrm{~h}$ at room temperature. Dark blue/green dispersions were obtained and subsequently characterized by UV-vis-NIR and ATR FT-IR spectroscopies. Derivatives 10-13 are fully water-soluble; thus, it was not necessary to use PSS or other stabilizers that are commonly used in the polymerization of EDOT. Herein, the anionic moieties on ETE-S and EEE-S effectively behave as the sulfonate groups of PSS, inducing self-doping. The positively charged groups on ETE-N and EEE-N are expected to act as cationic surfactants. Such cationic surfactants have already been successfully used to stabilize and dope PEDOT. ${ }^{20,26,27}$

The UV-vis-NIR absorption spectra of all polymeric dispersions are presented in Figure 1c, while photos of the 
dispersions are presented in Figure 1d. The successful polymerization is confirmed by the absence of the neat trimers absorption peaks between 300 and $400 \mathrm{~nm}$ (cf. Figure 2a). The appearance of broad absorption bands above $500 \mathrm{~nm}$ is consistent with the formation of conjugated polythiophene backbones. Specifically, for PEEE-S and PEEE-N, the broad vis-NIR absorption is assigned to both polaronic and bipolaronic states in the band gap, following the recent DFT-based interpretation reported by Zozoulenko et al. ${ }^{28}$ The more pronounced NIR absorption recorded for PEEE-S implies that this polymer exhibits a higher oxidation level than that of PEEE-N, corroborating the more efficient doping induced by the anion, in accordance with our initial hypothesis on the role of the cation. Concerning PETE-S and PETE-N, the broad absorption peaks centered at 700 and $780 \mathrm{~nm}$, respectively, are indicative of the polymers doping with bipolaron charge carriers. ${ }^{29}$ Once more, the higher absorption recorded for PETE-S above $1000 \mathrm{~nm}$ is consistent with a higher oxidation level of the anion-bearing polymer. For all four polymers, no peaks related to neutral chains are observed at around $550 \mathrm{~nm},{ }^{28,29}$ underlining the efficient doping of the polymers.

The conductivity of these new polymers in a thin-film configuration was measured using the four-point probe technique. The conductivities of PEEE-S and PEEE-N are 170 and $2 \mathrm{~S} \mathrm{~cm}^{-1}$, respectively, much higher than those of PETE-S and PETE-N (25 and $0.5 \mathrm{~S} \mathrm{~cm}^{-1}$ ), validating the hypothesis behind the design of EEE trimers, which suggests that all-EDOT trimers will conduct charges better than ETE trimers $^{30}$ due to enhanced planarity. Moreover, the sulfonatebearing trimers are more conducting than their cationic counterparts, consistent with their higher doping level. Note that the conductivities measured for the four trimers are higher than $0.2-0.35 \mathrm{~S} \mathrm{~cm}^{-1}$ values reported for PEDOT:PSS thin films without any additives, ${ }^{31-33}$ partially due to the absence of the electronically insulating PSS counterpart but also due to the self-induced doping in the case of the anionic-bearing trimers, or due to the indirect (medium-induced) doping, in the case of the cationic-bearing trimers.

Next, the in vitro enzymatic polymerization of the four trimers was evaluated. We have previously reported that the ETE-S trimer polymerizes efficiently in the presence of horseradish peroxidase (HRP) and hydrogen peroxide in physiological $\mathrm{pH} .{ }^{15}$ Here, the successful enzymatic polymerization of ETE-S synthesized following the novel synthetic route is achieved, as expected. The UV-vis spectrum of the reaction solution (ETE-S $360 \mu \mathrm{M}, \mathrm{H}_{2} \mathrm{O}_{2} 180 \mu \mathrm{M}$, HRP $5 \mathrm{U}$ $\mathrm{mL}^{-1}$ ) recorded after $5 \mathrm{~min}$ of incubation shows that the trimer ETE-S peak diminishes and a broad absorption band appears from $500 \mathrm{~nm}$ to NIR (Figure 2a), which corresponds to the doped state of PETE-S. ${ }^{29}$ When HRP or $\mathrm{H}_{2} \mathrm{O}_{2}$ are not included in the reaction solution, no polymerization occurs, as shown by the absorption signature of ETE-S at $350 \mathrm{~nm}$, which remains unaltered. Similar behavior was recorded for EEE-S (Figure 2b), which polymerizes only when both HRP and $\mathrm{H}_{2} \mathrm{O}_{2}$ are present in the reaction solution. The high absorption of the formed PEEE-S at NIR is assigned to polaronic and bipolaronic bands, as it was previously described for PEDOT, ${ }^{13,28}$ and confirmed the efficient self-doping of this polymer.

Concerning enzymatic polymerization of the two cationbearing trimers, the absorption spectra exhibit new peaks in the visible range that prove that polymerization does occur upon the addition of $\mathrm{H}_{2} \mathrm{O}_{2}$ and HRP. Nonetheless, the presence of the neat trimers signature at around $350 \mathrm{~nm}$ demonstrates that polymerization is not complete after $5 \mathrm{~min}$ of reaction, as not all trimers are consumed, and suggests that ETE-N and EEE-N are less reactive than ETE-S and EEE-S. This is probably driven by the lower solubility of these molecules in water, which can affect polymerization kinetics, slowing down the polymerization rate. Furthermore, this could arrive by a higher charge stabilization provided by the sulfonate group during polymerization.

Since absorption properties are highly defined by the backbone due to its high electron density, we move on and assign the ETE-N peaks based on the DFT calculations for ETE-S. ${ }^{29}$ The absorption peak at $500 \mathrm{~nm}$ matches very well the absorption from neutral (undoped) PETE chains, while that at $650 \mathrm{~nm}$ can be attributed to polaronic states of single oxidized oligomers or bipolaronic states formed in $\pi-\pi$ PETE stacks. Note that this later peak is blue-shifted with respect to the one predicted for PETE-S. We attribute this shift to the presence of the positive charge on the side chain since counterions are expected to affect the band position in the gap due to Coulomb interactions, ${ }^{28}$ besides, of course, the strong effect of oligomer length and chain packing on absorption. In contrast, Figure $2 \mathrm{~d}$ shows that EEE-N already polymerizes in the presence of $\mathrm{H}_{2} \mathrm{O}_{2}$, thanks to the presence of $\mathrm{OH}$ radicals. However, the formed polymer is in a reduced (neutral) state. When HRP is added in the reaction, a broad band centered at $700 \mathrm{~nm}$ appears, related to the doped state of the polymer. Similarly to ETE-N, this peak is blue-shifted with respect to the polaronic/bipolaronic peak predicted for PEDOT at $800 \mathrm{~nm}^{28}$ No NIR absorption is recorded. Therefore, enzymatically polymerized PETE-N and PEEE-N are found to be only partially doped after 5 mins of reaction and to exhibit a low oxidation level. Certainly, this is a result of the positively charged side chain that inhibits charge depletion from the backbone, favoring the undoped state. As already mentioned above, any charges in the backbone are then stabilized by anions present in the reaction solution. These findings highlight the efficient use of the side chain charge as a handle for tuning the oxidation level and, subsequently, conduction properties.

After the successful in vitro enzymatic polymerization of the four trimers, we proceed to evaluate their in vivo polymerization, using bean roots as the model system. A fresh root from a bean plant was immersed in a $1 \mathrm{mM}$ trimer solution for $24 \mathrm{~h}$. Then, the root was detached from the plant and observed under a stereomicroscope. In all cases, the trimers polymerize along the root, localized on the epidermis, forming a dark blue coating, in agreement with the in vitro experiments. Just like PETE-S, PEEE-S localizes on the epidermis without penetrating the internal structure of the root (Figure 2e,f). In the case of EEE-N and ETE-N, polymerization is observed not only on the epidermis of the root but also within the ground and vascular tissues. EEE-N polymerizes around the cell wall of the cortex cells as seen from the cross section (Figure $2 \mathrm{~h}$ ) while ETE-N crosses the pericycle and enters the vascular tissue (Figure $2 \mathrm{~g}$ ). The reason for the ability of these trimers to pass the epidermis and enter internally should be related to the functionalization of the conjugated backbones with a positively charged side chain. The in vitro experiments showed that the positively charged moieties slow down the polymerization process. Possibly, these slow kinetics allow the smaller and more mobile trimers to penetrate easier the plant's 
tissue before polymerizing. Moreover, the reduced water solubility of the cation-bearing trimers compared to the anion-bearing ones may drive the penetration of the former molecules deeper inside the root, where the environment is less aqueous. This hypothesis could be supported by the fact that ETE-N is less soluble than EEE-N and, thus, manages to reach the innermost of the root, and enters the vascular tissue.

In all cases, the trimers polymerize only on the part of the root that has been immersed in the trimer solution. Even in the cases where the trimers enter the internal structure of the root, no trimer is found in the part of the root that was not immersed in the solution, indicating, thus, that these trimers travel only laterally within the root and not longitudinally.

\section{CONCLUSIONS}

The synthesis of three new EDOT-based trimers that can be used as conducting building blocks in bioelectronics has been presented. The trimers can be chemically, electrochemically and enzymatic polymerized in vitro. Functionalizing the side groups with anions or cations proves to be an efficient way to tailor the doping level, as well as the oxidation potential, which consequently affects the polymerization kinetics. Furthermore, the trimers can be efficiently polymerized in vivo along the roots of living plants due to the presence of native peroxidase enzymes. The localization of the resulting polymer in the roots depends on the trimer structure. This work not only offers to the bioelectronics community a set of new water-soluble EDOT-based materials for tissue interface but, most importantly, draws design principles for the synthesis of selforganized conducting molecules for electronic functionalization of living tissue.

\section{EXPERIMENTAL SECTION}

Synthesis of the four trimers $(10,11,12$, and 13 in Figure 1a). The synthesis was performed using butane sultone and $N, N$-dimethylamino-2-chloroethane together with sodium hydride in anhydrous dimethylformamide (DMF). Then, iodomethane was used to quaternize the amine, obtaining the ammonium derivative. In all cases, the product is an insoluble powder and has been recovered by filtration. All details are provided in Supporting Information, Section 2. Details on polymerizations (chemical, electrochemical, in vitro and in vivo) as well as on characterization methods are provided in the Supporting Information.

\section{ASSOCIATED CONTENT}

\section{(s) Supporting Information}

The Supporting Information is available free of charge at https://pubs.acs.org/doi/10.1021/acsaelm.0c00861.

Materials and methods, synthesis and NMRs (dibromuration of 1 and 2; synthesis of EDOT boronic acid pinacol ester 5; Suzuki coupling reactions (trimerOHs) (6,7); ETE-S (10); EEE-S (11); ETE-N (12); EEE-N (13)); previous synthesis of ETE-S by Stavrinidou et al.; cyclic voltammetry; ATR FT-IR of chemically polymerized 14-17; films preparation and conductivity values; and AFM measurements (PDF)

\section{AUTHOR INFORMATION}

\section{Corresponding Authors}

Daniele Mantione - Laboratoire de Chimie des Polymères Organiques (LCPO-UMR 5629), Université de Bordeaux, Bordeaux INP, CNRS, F-33607 Pessac, France;
두 orcid.org/0000-0001-5495-9856;

Email: daniele.mantione@u-bordeaux.fr

Eleni Stavrinidou - Laboratory of Organic Electronics, Department of Science and Technology, Linköping University, SE-601 74 Norrköping, Sweden; Email: eleni.stavrinidou@ liu.se

Eleni Pavlopoulou - Laboratoire de Chimie des Polymères Organiques (LCPO-UMR 5629), Université de Bordeaux, Bordeaux INP, CNRS, F-33607 Pessac, France; (i) orcid.org/0000-0002-5291-0132; Email: epavlopoulou@enscbp.fr

\section{Authors}

Emin Istif - Laboratoire de Chimie des Polymères Organiques (LCPO-UMR 5629), Université de Bordeaux, Bordeaux INP, CNRS, F-33607 Pessac, France; Department of Mechanical Engineering, Koç University, 34450 Istanbul, Turkey; (1) orcid.org/0000-0003-4700-7050

Gwennael Dufil - Laboratory of Organic Electronics, Department of Science and Technology, Linköping University, SE-601 74 Norrköping, Sweden

Lorenzo Vallan - Laboratoire de Chimie des Polymères Organiques (LCPO-UMR 5629), Université de Bordeaux, Bordeaux INP, CNRS, F-33607 Pessac, France

Daniela Parker - Laboratory of Organic Electronics, Department of Science and Technology, Linköping University, SE-601 74 Norrköping, Sweden

Cyril Brochon - Laboratoire de Chimie des Polymères Organiques (LCPO-UMR 5629), Université de Bordeaux, Bordeaux INP, CNRS, F-33607 Pessac, France; (1) orcid.org/0000-0003-3242-1574

Eric Cloutet - Laboratoire de Chimie des Polymères Organiques (LCPO-UMR 5629), Université de Bordeaux, Bordeaux INP, CNRS, F-33607 Pessac, France; (1) orcid.org/0000-0002-5616-2979

Georges Hadziioannou - Laboratoire de Chimie des Polymères Organiques (LCPO-UMR 5629), Université de Bordeaux, Bordeaux INP, CNRS, F-33607 Pessac, France; - orcid.org/0000-0002-7377-6040

Magnus Berggren - Laboratory of Organic Electronics, Department of Science and Technology, Linköping University, SE-601 74 Norrköping, Sweden

Complete contact information is available at: https://pubs.acs.org/10.1021/acsaelm.0c00861

\section{Author Contributions}

"D.M. and E.I. contributed equally to this work.

\section{Notes}

The authors declare no competing financial interest.

\section{ACKNOWLEDGMENTS}

Dr. Gilles Pecastaings is warmly acknowledged for performing the AFM measurements. The Laboratoire de l'Intégration du Matériau au Système (IMS - UMR 5218) and Dr. Damien Thuau are warmly acknowledged for providing access to their UV-vis spectrometer. European Union is acknowledged for funding this research through Horizon 2020 research and innovation program under the grant agreement No. 800926 (HyPhOE) and the MSCA-IF-2018 No. 838171 (TEXTHIOL). 


\section{REFERENCES}

(1) Leger, J. M. Organic Electronics: The Ions Have It. Adv. Mater. 2008, 20, 837-841.

(2) Wang, X.; Shapiro, B.; Smela, E. Visualizing Ion Currents in Conjugated Polymers. Adv. Mater. 2004, 16, 1605-1609.

(3) Paulsen, B. D.; Tybrandt, K.; Stavrinidou, E.; Rivnay, J. Organic Mixed Ionic-Electronic Conductors. Nat. Mater. 2020, 1, 13-26.

(4) Simon, D. T.; Gabrielsson, E. O.; Tybrandt, K.; Berggren, M. Organic Bioelectronics: Bridging the Signaling Gap between Biology and Technology. Chem. Rev. 2016, 116, 13009-13041.

(5) Khodagholy, D.; Gelinas, J. N.; Thesen, T.; Doyle, W.; Devinsky, O.; Malliaras, G. G.; Buzsáki, G. NeuroGrid: Recording Action Potentials from the Surface of the Brain. Nat. Neurosci. 2015, 18, $310-315$.

(6) Khodagholy, D.; Doublet, T.; Gurfinkel, M.; Quilichini, P.; Ismailova, E.; Leleux, P.; Herve, T.; Sanaur, S.; Bernard, C.; Malliaras, G. G. Highly Conformable Conducting Polymer Electrodes for In Vivo Recordings. Adv. Mater. 2011, 23, H268-H272.

(7) Diacci, C.; Lee, J. W.; Janson, P.; Dufil, G.; Méhes, G.; Berggren, M.; Simon, D. T.; Stavrinidou, E. Real-Time Monitoring of Glucose Export from Isolated Chloroplasts Using an Organic Electrochemical Transistor. Adv. Mater. Technol. 2019, No. 1900262.

(8) Rivnay, J.; Inal, S.; Salleo, A.; Owens, R. M.; Berggren, M.; Malliaras, G. G. Organic Electrochemical Transistors. Nature Reviews Materials. 2018, 3, No. 17086.

(9) Guex, A. G.; Puetzer, J. L.; Armgarth, A.; Littmann, E.; Stavrinidou, E.; Giannelis, E. P.; Malliaras, G. G.; Stevens, M. M. Highly Porous Scaffolds of PEDOT:PSS for Bone Tissue Engineering. Acta Biomater. 2017, 62, 91-101.

(10) Richardson-Burns, S. M.; Hendricks, J. L.; Martin, D. C. Electrochemical Polymerization of Conducting Polymers in Living Neural Tissue. J. Neural Eng. 2007, 4, L6-L13.

(11) Liu, J.; Kim, Y. S.; Richardson, C. E.; Tom, A.; Ramakrishnan, C.; Birey, F.; Katsumata, T.; Chen, S.; Wang, C.; Wang, X.; Joubert, L. M.; Jiang, Y.; Wang, H.; Fenno, L. E.; Tok, J. B. H.; Paşca, S. P.; Shen, K.; Bao, Z.; Deisseroth, K. Genetically Targeted Chemical Assembly of Functional Materials in Living Cells, Tissues, and Animals. Science 2020, 367, 1372-1376.

(12) Liu, J.; Kim, Y. S.; Richardson, C. E.; Tom, A.; Ramakrishnan, C.; Birey, F.; Katsumata, T.; Chen, S.; Wang, C.; Wang, X.; Joubert, L. M.; Jiang, Y.; Wang, H.; Fenno, L. E.; Tok, J. B. H.; Paşca, S. P.; Shen, K.; Bao, Z.; Deisseroth, K. Genetically Targeted Chemical Assembly of Functional Materials in Living Cells, Tissues, and Animals. Science 2020, 367, 1372-1376.

(13) Stavrinidou, E.; Gabrielsson, R.; Gomez, E.; Crispin, X.; Nilsson, O.; Simon, D. T.; Berggren, M. Electronic Plants. Sci. Adv. 2015, 1, No. e1501136.

(14) Stavrinidou, E.; Gabrielsson, R.; Nilsson, K. P. R.; Singh, S. K.; Franco-Gonzalez, J. F.; Volkov, A. V.; Jonsson, M. P.; Grimoldi, A.; Elgland, M.; Zozoulenko, I. V.; Simon, D. T.; Berggren, M. In Vivo Polymerization and Manufacturing of Wires and Supercapacitors in Plants. Proc. Natl. Acad. Sci. U.S.A. 2017, 114, 2807-2812.

(15) Dufil, G.; Parker, D.; Gerasimov, J. Y.; Nguyen, T.; Berggren, M.; Stavrinidou, E. Enzyme-Assisted in Vivo Polymerisation of Conjugated Oligomer Based Conductors. J. Mater. Chem. B 2020, DOI: 10.1039/D0TB00212G.

(16) Jin, W.; Wang, R.; Huang, X. Horseradish Peroxidase-Catalyzed Oxidative Polymerization of Aniline in Bicontinuous Microemulsion Stabilized by AOT/SDS. J. Mol. Liq. 2020, 302, No. 112529.

(17) Liu, W.; Kumar, J.; Tripathy, S.; Senecal, K. J.; Samuelson, L. Enzymatically Synthesized Conducting Polyaniline. J. Am. Chem. Soc. 1999, 121, 71-78.

(18) Duan, L.; Zhao, Y.; Guo, F.; Liu, W.; Hou, C.; Ni, Z. Enzymatic-Catalyzed Polymerization of Water-Soluble Electrically Conductive Polymer PEDOT:PSS. Polym. Adv. Technol. 2014, 25, 896-899.

(19) Rumbau, V.; Pomposo, J. A.; Eleta, A.; Rodriguez, J.; Grande, H.; Mecerreyes, D.; Ochoteco, E. First Enzymatic Synthesis of Water-
Soluble Conducting Poly(3,4-Ethylenedioxythiophene). Biomacromolecules 2007, 8, 315-317.

(20) Bella, F.; Porcarelli, L.; Mantione, D.; Gerbaldi, C.; Barolo, C.; Grätzel, M.; Mecerreyes, D. A Water-Based and Metal-Free Dye Solar Cell Exceeding 7\% Efficiency Using a Cationic Poly(3,4-Ethylenedioxythiophene) Derivative. Chem. Sci. 2020, 11, 1485-1493.

(21) Istif, E.; Mantione, D.; Vallan, L.; Hadziioannou, G.; Brochon, C.; Cloutet, E.; Pavlopoulou, E. Thiophene-Based Aldehyde Derivatives for Functionalizable and Adhesive Semiconducting Polymers. ACS Appl. Mater. Interfaces 2020, 12, 8695-8703.

(22) Roncali, J.; Marque, P.; Garreau, R.; Gamier, F.; Lemaire, M. Structural Control of Conjugation in Functionalized Polythiophenes. Macromolecules 1990, 23, 1347-1352.

(23) Shi, L. H.; Gamier, F.; Roncali, J. Electrochemical and Chemical Syntheses of Poly(Thiophenes) Containing Oligo(Oxyethylene) Substituents. Macromolecules 1992, 25, 6425-6429.

(24) Lee, S.; Becht, G. A.; Lee, B.; Burns, C. T.; Firestone, M. A. Electropolymerization of a Bifunctional Ionic Liquid Monomer Yields an Electroactive Liquid-Crystalline Polymer. Adv. Funct. Mater. 2010, 20, 2063-2070.

(25) Casado, N.; Hernández, G.; Veloso, A.; Devaraj, S.; Mecerreyes, D.; Armand, M. PEDOT Radical Polymer with Synergetic Redox and Electrical Properties. ACS Macro Lett. 2016, 5, 59-64.

(26) del Agua, I.; Mantione, D.; Casado, N.; Sanchez-Sanchez, A.; Malliaras, G. G.; Mecerreyes, D. Conducting Polymer Iongels Based on PEDOT and Guar Gum. ACS Macro Lett. 2017, 6, 473-478.

(27) Mantione, D.; del Agua, I.; Sanchez-Sanchez, A.; Mecerreyes, D. Poly(3,4-Ethylenedioxythiophene) (PEDOT) Derivatives: Innovative Conductive Polymers for Bioelectronics. Polymers 2017, 9, No. 354.

(28) Zozoulenko, I.; Singh, A.; Singh, S. K.; Gueskine, V.; Crispin, X.; Berggren, M. Polarons, Bipolarons, And Absorption Spectroscopy of PEDOT. ACS Appl. Polym. Mater. 2019, 1, 83-94.

(29) Volkov, A. V.; Singh, S. K.; Stavrinidou, E.; Gabrielsson, R.; Franco-Gonzalez, J. F.; Cruce, A.; Chen, W. M.; Simon, D. T.; Berggren, M.; Zozoulenko, I. V. Spectroelectrochemistry and Nature of Charge Carriers in Self-Doped Conducting Polymer. Adv. Electron. Mater. 2017, 3, No. 1700096.

(30) Elschner, A.; Kirchmeyer, S.; Lovenich, W.; Merker, U.; Reuter, K.; Lövenich, W.; Merker, U.; Reuter, K. PEDOT: Principles and Application of an Intrinsically Conducting Polymer; CRC Press: Boca Raton FL USA, 2010.

(31) Xia, Y.; Ouyang, J. Significant Different Conductivities of the Two Grades of Poly(3,4-Ethylenedioxythiophene):Poly(Styrenesulfonate), Clevios P and Clevios PH1000, Arising from Different Molecular Weights. ACS Appl. Mater. Interfaces 2012, 4, 4131-4140.

(32) Yu, Z.; Xia, Y.; Du, D.; Ouyang, J. PEDOT:PSS Films with Metallic Conductivity through a Treatment with Common Organic Solutions of Organic Salts and Their Application as a Transparent Electrode of Polymer Solar Cells. ACS Appl. Mater. Interfaces 2016, 8, 11629-11638.

(33) Cruz-Cruz, I.; Reyes-Reyes, M.; Aguilar-Frutis, M. A.; Rodriguez, A. G.; López-Sandoval, R. Study of the Effect of DMSO Concentration on the Thickness of the PSS Insulating Barrier in PEDOT:PSS Thin Films. Synth. Met. 2010, 160, 1501-1506. 